Conference Presentation

\title{
A systematic review about animal models in homeopathic research: the last five years of PubMed indexed papers
}

\author{
Leoni Villano Bonamin, Thayna Neves Cardoso, Aloisio Cunha Carvalho, Juliana G Amaral \\ Centro de Pesquisa, UNIP, São Paulo, Brazil, leonibonamin@gmail.com
}

\begin{abstract}
Background: This review is part of a special issue of Journal "Homeopathy" (ELSEVIER) to be published in 2015, about the follow-up of researches published in the book titled "Ultra-High Dilution, Physiology and Physics", written in 1994 and edited by PC Endler and J Schulte. In 2010 a critical and conceptual review about animal models in homeopathy and high dilution research was published by our group. Thus, the aim of this study is to know how models and conclusions have progressed in this field in the last five years. Animal models represent one of the most illustrative examples of the biological effects of homeopathy and high dilutions. The main contribution of these studies is the comprehension of biological features and the phenomenology towards the high dilutions effects upon living systems, which represents an important step to the understanding of mechanisms of action of homeopathic medicines.
\end{abstract}

Methods: 53 articles indexed in the PubMed database were systematically evaluated, in which 12 different animal species were used. 29 out of 53 reported studies were performed with "ultra-high" dilutions, whereas 14 studies were performed using dilutions in the range or below $10^{-23}$ or with commercial complexes (10).

Results: Only 02 negative results were reported, both using commercial complexes as tested medicine (one each in fish and bee model). Since 2010, the quality of employed methods, mainly statistics, has improved. The inclusion of more refined protocols, such as in vitro primary cell cultures and ex vivo protocols (10/53), often with three or more repetitions, could be observed. These new methods allowed to observe epigenetic mechanisms involved.

Conclusion: The demonstration of biological effects of homeopathy on animal models, in the past 5 years, is more refined than those previously described, helping in the development of paths to the mechanism of action discovery.

Keywords: Homeopathy, high dilutions, animal models, systematic review

(C) International Journal of High Dilution Research.

Not for commercial purposes.

OPEN ACCESS

Cite as: Bonamin LV, Cardoso TN, Carvalho AC, Amaral JG. A systematic review about animal models in homeopathic research: the last five years of PubMed indexed papers. Proceedings of the XXIX GIRI Meeting; 2015 June 3 - 5; Verona (Italy). Int J High Dilution Res. 2015; 14(2): 45-45 